\title{
SOBRE LA ASPIRACIÓN Y ELISIÓN DE /S/ IMPLOSIVA Y FINAL EN EL ESPAÑOL DE PUERTO RICO
}

El español hablado en Puerto Rico es una de las variedades mejor conocidas del orbe hispánico y ello gracias a los esfuerzos de un gran fonetista: Tomás Navarro Tomás. Tan detallado y completo es su estudio ${ }^{1}$ que sólo después de notables avances tecnológicos han podido superarse y extenderse tanto cualitativa como cuantitativamente sus observaciones.

Fue precisamente para aprovechar esos avances que se propuso el "Proyecto coordinado de la norma lingüística culta de las principales ciudades de América Latina y de España" 2, cuya meta principal es la recolección de muestras del habla culta en cinta magnetofónica para su posterior análisis. Los resultados de este tipo de investigación en el campo de la dialectología se lograrán al realizar un análisis completo y minucioso del habla natural.

Paralelamente con los avances técnicos en la grabación del habla se han producido grandes innovaciones en la teoría fonológica. El enfoque generativo ${ }^{3}$ provee una metodología para el examen de los llamados "procesos fonológicos", los cuales producen las variantes fonéticas tradicionalmente estudiadas por los dialectólogos. W. Labov en sus estudios de dialectos del inglés ${ }^{4}$ ha desarrollado una

1 Tomás Navarro Tomás, El español en Puerto Rico, Río Piedras, 1948.

2 Noticia más detallada de este proyecto puede encontrarse en el informe de Juan M. Lope Blanch en las Actas, informes y comunicaciones del Simposio de México, Universidad Nacional Autónoma, México, 1969, pp. 222-233.

3 Prefiero la versión "natural" del modelo generativo que puede consultarse en Joan B. Hooper, Aspects of natural generative phonology, Academic Press, 1977. Para una discusión amplia de la influencia del método generativo en los estudios dialectales, véase mi artículo "Dialectología española" en J. Guitart y J. Roy, eds., La teoria generativo-transformacional y la gramítica española, Barcelona (en prensa).

4 Sociolinguistic patterns, Philadelphia, 1973, es un resumen de gran parte de su trabajo hasta esa fecha. 
metodología para el estudio de la variación fonológica dialectal, de la cual ha surgido un nuevo modelo analítico. Este modelo teórico, a veces llamado de variabilidad sociolinguiistica, se ha combinado con una metodología de recolección de datos directamente del habla grabada en tres investigaciones extensivas de fenómenos dialectales hispánicos. La primera es el admirable estudio del español de emigrantes puertorriqueños en Nueva York de Elenor Ma y Roxana Herasimchuk ${ }^{5}$ quienes llevaban como propósito principal la investigación de este problema: ¿Cómo funcionan las múltiples variedades de la lengua (o lenguas) para llenar las distintas necesidades de comunicación en una comunidad? Con ese fin definieron una serie de "estilos" del habla: habla informal, habla formal, producción oral de palabras elicitadas, lectura oral de un texto y lectura oral de una lista de palabras preseleccionadas. Intentaron así establecer la correlación entre la variación fonológica y esta escala de medida estilística.

Otra investigación en la cual se analizó el habla según el modelo de Labov es el estudio monumental de Henrietta Cedergren sobre el español de Panamá ${ }^{6}$. Este trabajo es el más completo y riguroso de la variación fonológica de un dialecto hispánico hasta la fecha, y ha servido de estímulo para este artículo. Por lo demás, los únicos estudios de variabilidad fonológica basados en el modelo de reglas fonológicas variables para los cuales se utilizaron los materiales del proyecto de la norma culta son los que escribí sobre el español cubano?.

5 "The linguistic dimensions of a bilingual neighborhood" en Joskua Fishman, Bilingualism in the Barrio, Bloomington, Indiana, 1972.

${ }_{6}$ The interplay of social and linguistic factors in Panama, tesis, Cornell University, 1973.

7 "La aspiración y elisión en el español cubano. Implicaciones para una teoría fonológica dialectal", ponencia, IV Congreso Internacional del ALFAL, Lima, 1975; "Functional constraints on deletion of word final /s/ in Cuban Spanish", B. L. S., t. 1, pp. 431-437, Berkeley, 1975; "Natural generative phonology: Evidence from Spanish", Proceedings of the Pacific Northwest Conference on foreign languages, Vancouver, 1975; "La nasal implosiva y final en el español cubano", $A L M, 13,1975,257-271$; "The inherent variability of word final /s/ in Cuban and Puerto Rican Spanish", Teaching Spanish to the Spanish speaking, G. Valdés Fallis y R. García Moya, eds., San Antonio, Texas, 1976; "Paralleslisms between liaison in French and /s/ aspiration and deletion in Spanish dialects", Proceedings of the Sixth Linguistcs Symposium on Ro. mance Languages, Montreal, April, 1976; "The relationship between the group and the individual in the analysis of variable rules. Evidence from Spanish", Berkeley Linguistics Society, t. 3, 1977. Están en preparación: "The aspiration and deletion of word final /s/ in Cuban Spanish"; "The aspiration and deletion of final /s/ in Cuban and Puerto Rican Spanish". Son importantes los estudios de Clara Wolf y Elena Jiménez presentado en Lima, al con- 


\section{MÉTOdos Y MATERIALES}

Para investigar la variación fonológica en el español de Puerto Rico, se seleccionaron como muestra dieciséis de las entrevistas grabadas. Los investigadores ${ }^{8}$ del proyecto en Puerto Rico han seguido fielmente las normas establecidas por la Comisión General para la selección de informantes. Todos, sin excepción, son de nivel socioeconómico superior, graduados universitarios, por lo menos en licenciatura, y todos residentes urbanos. La edad de los informantes varía entre los veinticinco y los ochenta años. Cada entrevista duró de treinta minutos a una hora. Generalmente, el informante habló sobre un tópico seleccionado de antemano lo que llevó a una entrevista semiformal, pero no forzada. Así pues, los resultados que ofrezco aquí representan 'el sistema fonológico del nivel culto, no del habla popular de Puerto Rico.

El primer paso del análisis consistió en seleccionar las variantes fonológicas para transcribir. La representación fonética de la /s/ final registra una gran variación, aspecto sobre el que ya había tratado Navarro Tomás. Tanto Ma y Herasimchuk como Cedergren decidieron distinguir solamente tres variantes principales; tomé también esta decisión después de intentar infructuosamente distinguir sistemáticamente entre más variantes en el análisis de las entrevistas cubanas. Las tres variantes principales que normalmente se distinguen para /s/ son: $s$ : fricativo alveolar sorda-sibilante; $h$ : aspiración faríngea, a veces sonorizada, a veces muy asimilada a la consonante siguiente; $\phi$ : cero fónico, ausencia total del segmento.

El segundo paso fue la transcripción fonética de todos los casos de /s/ final que se presentaron al escuchar las entrevistas. La tarea

greso del PILEI en 1975, "El yeísmo rioplatense"; B. Fontanella de WeinBERG, "Comportamiento ante -s de hablantes femeninos y masculinos del español bonaerense", $R P h, 27$ (1972), 50-58. También los lingüistas mexicanos han utilizado los materiales del proyecto en varios estudios de carácter fonológico. Desde que se escribió este informe también ha completado su trabajo Beverly Jean Longmire sobre el español de Mérida, Venezuela, The relationship of variables in Venezuelan Spanish to historical sound changes in Latin and the Romance languages, tesis, Georgetown University, Washington, D. C., 1976.

8 Quisiera reiterar mi profundo agradecimiento al profesor Humberto López-Morales, director del proyecto en Puerto Rico, a la profesora Amparo Walters, y a todos los investigadores de este proyecto que han colaborado tan magnificamente con nosotros. También agradezco mucho al profesor Jorge Guitart, por sus sugerencias valiosas en la preparación de la versión final de esta monografía.

9 He tratado este asunto con más detalle en mi artículo "La aspiración en el español de Cuba. Observaciones teóricas", $R L A, 13$ (1975), 93-107. 
de la transcripción fonética de todas las ocurrencias de / $/$ final se dividió entre cuatro ayudantes expertos ${ }^{10}$. Cada transcripción fue revisada por otro ayudante y finalmente por el investigador principal, de manera que para cada entrevista las transcripciones se verificaron tres veces. Las entrevistas produjeron un total de más de 20,000 casos de /s/ final.

El tercer paso consistió en pasar la información sobre el fonema y sus manifestaciones fonéticas con su contexto fonológico y gramatical, a tarjetas IBM para su subsecuente tabulación. En este estudio aprovechamos toda la experiencia de las investigaciones previas. Por ejemplo, Ma y Herasimchuk habian constatado que el contraste "vocal-consonante" para el segmento que sigue a /s/ es de gran importancia. Cedergren y Terrell habian verificado por separado que la diferenciación entre posición implosiva (ante consonante) y la posición final absoluta (ante pausa) es indispensable para describir el sistema de variación fonológica de $/ \mathrm{s} /$. Al encontrar dificultades en una parte de su análisis, Cedergren decidió tomar una submuestra de sus datos para verificar el efecto de posición del acento en palabras que empiezan con vocal, correlación que resultó ser positiva.

Dados estos antecedentes, decidimos incluir cuatro contextos fonológicos de importancia potencial en el condicionamiento fonológico de la /s/ final de palabra: implosiva: los niños; final absoluta: los niños; ante vocal acentuada: los hijos; ante vocal no acentuada: los inteligentes. Además, en los estudios de $\mathrm{Ma}$ y Herasimchuk, Cedergren, y Terrell se comprobó que el fenómeno de aspiración y elisión de /s/ está condicionado sistemáticamente por factores morfológicos y gramaticales. Por eso en esta investigación se decidió codificar toda información morfológica o gramatical que pudiera tener alguna influencia en estos procesos fonológicos.

Primero, se diferenció entre /s/ como morfema gramatical (de pluralidad o verbal: niños o tienes) y /s/ de palabras en las cuales no es un morfema gramatical (vez, luz, entonces, etc.). La /s/ de estas palabras, que denominaré $s$ léxica, se dividió en las siguientes subcategorías: 1) entonces, 2) pues, 3) más, 4) nombres propios, 5) otras palabras. La /s/ gramatical se dividió primero en morfema de pluralidad y morfema verbal. Como morfema verbal hay tres posibilidades: 1) final del morfema de la primera persona del plural (hablamos); 2) segunda persona del singular (tienes); 3) forma es del verbo ser. La /s/ del plural se considera en el adjetivo (bonitos) en el sustantivo (nombres), o en el pronombre (ellos).

10 Agradezco mucho su participación y largas horas de transcripción a James D. Davis, Jean Egasse, Barbara Reid y Deborah Ross. 
Se diferenciaron cuidadosamente los múltiples casos de modificadores puesto que nos interesó averiguar el papel desempeñado por la redundancia sintáctica y morfológica: 1) primer modificador (los tres libros; 2) modificadores subsecuentes antepuestos (los primeros libros); 3) modificadores pospuestos (los libros interesantes); 4) modificador precedido de cópula (son interesantes).

El cuarto paso fue la utilización de la computadora para clasificar las transcripciones fonéticas atendiendo a los criterios ya señalados. Para estos fines se utilizó el subprograma CROSSTAß3S del llamado SPSS (Statistical Package for the Social Sciences) ${ }^{11}$, programa que produce cuadros de dimensiones $n$ por $m$; se puede así obtener cuadros de datos en los que se relacionan las variantes con sus respectivos contextos, lo que produce más de 200 cuadros de datos empíricos. El análisis siguiente se basa en estos datos.

\section{/S/ INTERIOR Y FINAL}

La diferencia entre posición interior y posición final es significante en el caso del tratamiento del fonema /s/. En el siguiente cuadro se comparan los índices de elisión, retención y aspiración según la posición de $/ \mathrm{s} /$ en la palabra.

I. /S/ INTERIOR Y FINAL

Elisión de /s/

Retención de /s/

en forma de:

(1) sibilante

(2) aspiración

Número de casos

Interna
$5 \%$

$3 \%$
$92 \%$
5951

Interna

5951
Final

$29 \%$

$15 \%$

$56 \%$

14393

En posición final de sílaba interna la norma entre estos informantes es la aspiración; los índices de elisión o aspiración tienen importancia reducida. En cambio, en posición final de palabra la variabilidad en la pronunciación del fonema /s/ es impresionante: aumenta tanto la elisión del fonema que el índice representa casi la tercera parte de los casos examinados, mientras que la conservación de alófonos sibilantes, también aumenta, aunque no a tal grado como en el caso de la elisión.

11 Véase N. Nie, D. Bent, C. H. Hull, S. P. S. S., 1970. Agradezco muchísimo la colaboración del centro computacional de la Universidad de California, Irvine, y especialmente a Karen Anderson y Cathy Smith quienes trabajaron muchas horas para resolver problemas técnicos. 


\section{Condicionamiento Fonológico general}

Desde el punto de vista puramente fonológico podríamos haber esperado una diferencia entre el resultado de /s/ en posición interior y el mismo fonema en posición final de palabra puesto que la /s/ implosiva interior forzosamente está antes de una consonante, mientras que en el caso de /s/ final de palabra el contexto fonético es más complicado dado que puede seguirle una consonante, una vocal o una pausa; es decir, puede encontrarse en posición implosiva o explosiva prevocálica. Así, es probable que por lo menos en parte la explicación de las diferencias en el tratamiento de /s/ interior y final de palabra se encuentre en un examen detallado de los índices de elisión, retención y aspiración según estos varios contextos fonológicos.

II. / $/$ INTERIOR Y FINAL SEGÚN EL CONTEXTO FONOLÓGICO

$\begin{array}{lcccc} & \text { Elision } & \begin{array}{c}\text { Retención } \\ \text { con sibilante }\end{array} & \begin{array}{c}\text { Retención con } \\ \text { aspiración }\end{array} & \begin{array}{c}\text { Numero de } \\ \text { casos exa- } \\ \text { minados }\end{array} \\ \begin{array}{l}\text { Interior } \\ \text { ante consonante }\end{array} & 5 \% & 3 \% & 92 \% & 5951 \\ \begin{array}{l}\text { Final ante } \\ \text { consonante }\end{array} & 25 \% & 2 \% & 73 \% & 7239 \\ \begin{array}{l}\text { Final ante } \\ \text { vocal }\end{array} & 31 \% & 18 \% & 50 \% & 3604 \\ \text { Final ante pausa } & 33 \% & 40 \% & 27 \% & 3539\end{array}$

La elisión del fonema es mucho más frecuente en posición final de palabra que en posición final de sílaba interior. En posición interior y final ante consonante los índices de elisión son muy distintos, lo cual nos obliga a buscar otra explicación distinta a la del contexto fonológico. En el caso de la retención de la sibilante, en cambio, hay una correspondencia exacta entre el bajo índice de sibilancia ante consonantes, tanto en posición interior como final $(3 \%$ y $2 \%$ respectivamente). Aunque la pronunciación del fonema con un alófono sibilante no es la norma estadística en ningún contexto fonológico se ve claramente en el cuadro que la presencia de una vocal siguiente favorece la sibilante ligeramente y el encontrarse en posición final absoluta favorece fuertemente la sibilante más que cualquier otro alófono.

En resumen, podemos decir que el contexto fonológico de la /s/ determina en parte su manifestación alofónica. La presencia de una consonante siguiente impide claramente la realización sibilante del fonema y favorece la aspiración. La presencia de una vocal siguien- 
te admite la sibilancia pero favorece la aspiración. La posición final absoluta favorece la articulación del fonema en forma sibilante y limita la aspiración, aunque no es desconocida pues de ninguna forma se siente raro oír este alófono en esta posición.

La elisión total del fonema parece ser un poco más difícil de explicar. Según los datos del cuadro II, el índice se mantiene más o menos estable a un nivel de aproximadamente un cuarto a un tercio del número total de casos en todos los contextos fonológicos. Esta variación, aunque a ciertos niveles estadísticamente relevante, no parece ser lingüísticamente significante ${ }^{12}$. Así, la sistematización y explicación de la elisión tendrá que hacerse basándose en otros criterios además de los fonológicos.

\section{ELISIÓN DE /s/}

Es lógico pensar que el proceso de elisión de /s/ pudiera estar condicionado por factores morfológicos y sintácticos a causa de sus

$12 \mathrm{La}$ determinación de "significancia", sea ésta solamente al nivel estadístico o al nivel lingüístico, no es fácil. Al aumentar mucho el número de casos examinados, hasta una diferencia pequeña en el índice de aplicación resultará significante, estadísticamente hablando. Sin embargo, esto no indica que, forzosamente, la diferencia sea también significante a nivel lingüístico. En mi artículo para la Berkeley Linguistic Society, cit. supra, nota 7 , traté este problema y llegué a la conclusión de que en casos dudosos donde hay significancia estadística, se duda de la significancia lingüística; hay que examinar el comportamiento de cada uno de los informantes individuales. En este caso particular, al aplicar a estos 'datos tests de confianza de intervalos (al nivel de .99 de confianza), resulta una diferencia estadística importante sólo en el caso de la posición preconsonántica: $25 \%(23 \%-27 \%), 31 \%(29 \%-33 \%)$, $33 \%(31 \%-35 \%)$. Esto podría explicarse en términos del método de trabajo. Al transcribir /s/ elidida, tratamos de ser conservadores y no usar el símbolo $\phi$ si no estábamos totalmente seguros de que no había ningún rasgo de /s/ en la enunciación de la palabra. Cuando a /s/ le sigue una vocal o una pausa, esto resulta relativamente fácil, pero en el caso de $/ \mathrm{s} /$ más consonante es muy difícil distinguir entre una / $/$ / aspirada débilmente o una asimilación completa (resultado de una geminación de consonantes, que también se transcribió $h$ ). Así, creemos que el índice ligeramente más bajo en esta posición se debe totalmente a nuestra transcripción y no a una supuesta diferencía real en la conciencia del hablante puertorriqueño. La variación individual también apoya nuestra conclusión, ya que no hubo ninguna concordancia entre los informantes en cuanto a la elisión en los varios contextos fonológicos y concluimos que la variación se debe a que en el nivel individual no hay suficientes casos para que se vea siempre la regularidad. En el estudio del español porteño el contexto fonológico sí tuvo un efecto grande en la operación de la elisión de /s/ final (cf. mi artículo "La aspiración y la elisión de la /s/ final en el español porteño", Univ. de Calif., Irvine, 1977; mecanogr.). 
múltiples funciones en español. Como hemos dicho, /s/ final tiene tres funciones principales: representar el morfema de plural, ser parte integrante de una palabra (lo que llamamos la /s/ léxica) y ser parte de morfemas o formas verbales. Su función léxica es bastante simple, ya que con pocas excepciones la presencia o ausencia de una /s/ final no afecta la comprensión de la palabra: entonce(s), despué(s), lu(z), análisi(s), etc. Aun en los pocos casos de homofonía el contexto es suficiente para evitar posible confusión: Él se ve muy bien, una ve(z) estuvo aqui ella. La función de /s/ verbal es más complicada porque puede ser simplemente léxica, en $e s$ (la confusión con la conjunción $e$ es más teórica que real), formar parte del morfema de la primera persona del plural /-mos/, y representar todo el morfema de la segunda persona del singular familiar: tú tiene(s). Pero en la práctica la presencia del pronombre $t u ́$ o el contexto general siempre aclara la ausencia de $/ \mathrm{s} /$.

El caso de /s/ plural presenta serias complicaciones. El sistema morfosintáctico del español requiere la concordancia de número dentro de la frase nominal. De allí resultan múltiples representaciones, a veces redundantes, del morfema de plural. Sin embargo, algunas de estas representaciones no son redundantes, sino claves para la comprensión de la pluralidad por medio de su representación morfológica.

Hay varias posibilidades para la interpretación de la pluralidad. Podría interpretarse totalmente del contexto del habla sin ningún indicador morfológico. Un caso menos extremo para el español sería la interpretación según el contexto y según ciertos indicadores morfológicos que no sean /s/, por ejemplo, por medio de la concordancia verbal: son la(s) suya(s); por medio de la concordancia de número de ciertos adjetivos que terminan en una consonante en su forma singular: allí encontrarás la(s) parte(s) fácile(s); o de cambios vocálicos de ciertos determinadores: lo(s) libro(s).

Veremos que el habla culta de San Juan, por lo menos en esta etapa de desarrollo, ha rechazado estas posibilidades a favor del mantenimiento de un morfema de pluralidad representado o por la sibilante o por la aspiración según el contexto fonológico, en ciertas posiciones en frase nominal ${ }^{13}$. Sin embargo, una vez pronunciado el fonema /s/ de plural en la frase nominal, existe una tendencia muy fuerte a suprimir totalmente las otras representaciones de /s/ sintácticamente redundantes.

Examinaremos en algún detalle la operación de la elisión de /s/ léxica, verbal y plural siguiendo este orden.

13 Hay datos del trabajo de Cedergren (por medio de comunicación personal), de Shana Poplack sobre los puertorriqueños de Philadelphia y de Maxi- 
ELISIÓN DE /s/ LÉXICA

En esta investigación se dividió el total de casos de /s/ léxica en cinco categorías: monosílabos (luz, vez, tos, voz); polisílabos (antes, después, crisis, ciprés, lápiz); y las palabras entonces, más, y pues. Es posible que una mayor subcategorización arrojara resultados algo diferentes ya que es en esta categoría donde se esperaría mucha variación. Sería fácil que un hablante en una etapa avanzada de elisión reestructurase la forma fonémica de alguna palabra específica $\sin / \mathrm{s} /$ final. Sospechamos que esto es precisamente lo que ha pasado en algunos con la palabra entonces, que aparece muy frecuentemente en la forma [entonse].

III. ELISIÓN DE / / LÉXICA

$\begin{array}{lr}\text { entonces } & 70 \% \\ \text { pues } & 31 \% \\ \text { nombres } & 28 \% \\ \text { polisílabos } & 20 \% \\ \text { monosílabos } & 8 \% \\ \text { más } & 4 \%\end{array}$

En general, la /s/ de los monosílabos se elide muy poco con la excepción de pues. Las demás palabras sufren elisión normal, o sea entre la cuarta y la tercera parte de los casos, excepto entonces, la cual, como dijimos, parece haber sido reestructurada por algunos informantes $\sin / \mathrm{s} /$.

\section{ELISIÓN DE /s/ verbAL}

Pensábamos que el caso de la forma verbal es sería tratado igual a cualquier forma léxica monosilábica y, efectivamente, así fue, por lo menos entre los informantes de esta investigación. La elisión total de /s/ en es es baja, siendo más normal la aspiración o la realización sibilante según el contexto fonológico. Frente a esto, la /s/

miliano Sahater (República Dominicana) de que en otros estratos sociales el índice de elisión ha llegado a un extremo de aplicación, de modo que las posibilidades que mencioné pueden resultar acertadas para esos informantes. La verificación de esta posibilidad será de mucho interés ya que podría demostrarse cómo dos grupos de hablantes pueden operar con un sistema gramatical muy distinto sin 'problemas de comunicación. También sugiere otro análisis en que ciertos hablantes reestructuran su gramática y operan con una regla de insersión de /s/ en vez de una de elisión. 
redundante del morfema /-mos/, siempre polisilábico, se elide muy frecuentemente.

En el caso de /s/ correspondiente a $t u$ pronominal, el tratamiento está condicionado por el número de sílabas: la elisión de /s/ verbal en monosílabos (ves, das, vas) es muy poco común, pero es frecuente en formas polisilábicas.

IV. ELISIÓN DE / / VERBAL

$\begin{array}{lr}\text {-s, monosilábica }(\simeq \text { tú) } & 4 \% \\ \text { es } & 16 \% \\ -\operatorname{mos} & 48 \% \\ -s, \text { polisilábica }(\simeq \text { tú }) & 53 \%\end{array}$

Así, el tratamiento de /s/ verbal parece corresponder al mismo tratamiento de /s/ léxica en el que el número de sílabas es el factor que rige la elisión. Esperábamos que el factor de contraste en el caso de /s/ de segunda persona singular jugara un papel condicionante en su retención. Sin embargo, no existe tal condicionamiento, y la /s/ verbal parece tratarse como cualquier/s/ léxica. Sin duda, este tratamiento de /s/ de la segunda persona del singular está relacionado con la notable tendencia en el español del Caribe a usar las formas pronominales, sobre todo el pronombre tú.

\section{Elisión de /s/ PLURAL}

Para examinar la elisión en la frase nominal, se dividió el número total en las siguientes categorías: 1) /s/ de determinativos y otros adjetivos que aparecen primeramente en la frase nominal: lo(s) otros muchachos; 2) /s/ de adjetivos en segunda posición en la frase nominal que anteceden el sustantivo modificado: los primero(s) libros; 3) /s/ de adjetivos que siguen al sustantivo modificado: mis atentados infructifero(s); 4) adjetivos que siguen a un verbo: son inteligente(s); 5) pronombres contrastivos con $/ \mathrm{s} /:$ ello $(s)$, ella(s), lo(s), la(s), le(s) (éstas como complemento), no(s); 6) pronombres con /s/ redundante; nosotro(s); y 7) sustantivos: profesore(s).

\section{ELISIÓN DE /s/ PLURAL}

Primera posición

Pronombres no redundantes

Segunda posición del adjetivo

Adjetivo redundante

Pronombres redundantes (nosotros)

Nominal

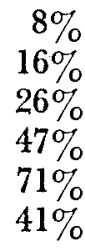


Las diferencias en los casos extremos es significativa: el adjetivo en primera posición con un índice de elisión de $8 \%$ contrasta con los adjetivos con /s/ redundante con un índice de elisión de 47\%. La /s/ de los pronombres contrastivos se elide en un $16 \%$ lo cual contrasta con el pronombre nosotros en el que /s/ es totalmente redundante, con una elisión de un $71 \%$. Los adjetivos en segunda posición ocupan una situación intermedia $(26 \%)$ entre el índice bajo de elisión (8\%) de los de primera posición y el índice alto de los que llevan /s/ redundante $(47 \%)$. Así, grosso modo puede concluirse que el puertorriqueño culto varía la elisión de /s/ plural de manera que normalmente conserva un indicador de pluralidad morfémica en los determinativos (sea alofónicamente un sibilante o una aspiración) pero la elide libremente en los casos en que /s/ es redundante por concordancia.

Cabe preguntar si hay otros factores que influyen en la aplicación de la regla de elisión en estos casos. Por ejemplo, sería lógico encontrar una diferencia entre la elisión de / $/$ / en las (casas) que la de /s/ en los (libros), ya que en éste existe el cambio $e l \rightarrow l o$, que pudiera indicar pluralidad. Sin embargo, los informantes de este estudio no utilizaron este posible condicionamiento morfológico. El índice de elisión de /s/ de las fue un $7 \%$ mientras el de los fue un $5 \%$, el opuesto a lo esperado, aunque la diferencia no es significante ni estadística ni lingüisticamente. También cabe preguntarnos si el factor del número de sílabas influye en la elisión de /s/ plural. Esto no es muy fácil de determinar, ya que en los casos de elisión alta, los adjetivos y sustantivos plurales son casi sin excepción polisílabas. Hay solamente dos posibilidades de comprobar la influencia del número de sílabas. En el caso de ellos, podríamos esperar que, por ser contrastivo /s/ (ello-ellos; ella-ellas), el indice de elisión fuera 'relativamente bajo. Por otra parte, por ser polisílaba podríamos suponer tal vez un aumento en frecuencia de elisiones. Al comparar la elisión de /s/ de ellos, ellas ' $13 \%$ ) con la de los, las, les (complemento) (17\%), o con la de nos (19\%), todos pronombres monosílabos, vemos que el número de sílabas aparentemente no afecta en absoluto la elisión de esta /s/. Otro caso es el de los determinativos (excluyendo los casos de los artículos los y las). Algunos son monosílabos (mis, tus, sus) y otros polisílabos (otros, muchos, estos). Si el factor del número de sílabas juega un papel en la elisión de /s/ plural, debe de suprimirse más frecuentemente la /s/ de los polisílabos. Esto no resulta ser el caso, ya que en los monosílabos la elisión fue de un $14 \%$ y en los polisílabos de un $10 \%$, diferencia estadísticamente no significante.

En resumen, parece que la elisión de /s/ plural se rige por un solo factor: el de la redundancia de /s/ en la frase nominal. 


\section{RETENCIÓN DE /S/: ASPIRACIÓN Y SIBILANCIA}

Hemos visto que la elisión de /s/ se ve condicionado por dos factores: la redundancia en el caso de /s/ plural y el número de sílabas en los demás casos. No hubo condicionamiento fonológico significante. En cambio, vimos parcialmente (cuadro 2) que la selección entre la retención del fonema en términos de sibilancia o de aspiración parece ser una cuestión regulada totalmente por el contexto fonológico. Los factores funcionales o gramaticales no parecen tener ya un papel en este fenómeno ${ }^{14}$. Esto es lógico si consideramos que la opción entre aspiración o sibilancia no afecta en absoluto la presencia del fonema o el significando del enunciado como puede suceder en el caso de la elisión. Así, aspirar o conservar la sibilante puede explicarse mejor en términos del contexto fonológico $\mathrm{y}$ en términos de preferencia y gustos personales. Por esta razón hay más variación individual (y dialectal) en el uso de alófonos aspirados o sibilantes que en el caso de la elisión. En la discusión siguiente se usará el índice de retención de un alófono sibilante para medir esta selección.

\section{RETENCIÓN DE SIBILANTE SEGÚN EL CONTEXTO FONOLÓGICO}

Interior ante consonante

Final ante consonante

Final ante vocal

Final ante pausa

La sibilante se presenta más en posición final absoluta, es decir, ante pausa. En esta posición es el alófono más común $40 \%$

14 Es posible probar que los factores morfológicos o funcionales no influyen en la selección entre aspiración o sibilante, pero se extendería mucho este informe ya que los datos son bastante complicados. Doy sólo algunos ejemplos: hay cierta variación al comparar la retención de /s/ como sibilante en las siguientes clases gramaticales: adjetivo en primera posición (10\%), pronombre $(10 \%)$, palabras léxicas $(20 \%)$, sustantivos $(17 \%)$, adjetivos con /s/ redundante $(15 \%)$. Sin embargo, esta variación no se debe a diferencias gramaticales sino a que ciertas clases gramaticales son más frecuentes que otras en ciertos contextos fonológicos. Por ejemplo, el uso de determinados en posición final absoluta es muy raro (de ahí el bajo nivel de sibilantes en esa categoría). La posible influencia del número de sílabas es más difícil de determinar. En general parece haber una ligera tendencia a usar sibilantes más en las palabras monosilábicas que en las polisilábicas. Sin embargo, la diferencia no es estadísticamente importante en la gran mayoría de los casos y no creo que sea lingüísticamente significante en ninguno de ellos. 
sibilante, $33 \%$ elisión, $27 \%$ aspiración). La posición implosiva, en cambio, favorece mucho la aspiración (final de palabra, $73 \%$ aspiración, $25 \%$ elisión, $27 \%$ sibilante), y hasta podría afirmarse que la sibilante en esta posición se siente anormal, ya que es un indicador de énfasis o de otras circunstancias especiales. En posición prevocálica existe la posibilidad de una sibilante, pero más común es la aspiración (50\% aspiración, $31 \%$ elisión, $18 \%$ sibilante). Es probable que al adoptar el proceso de aspiración, éste se haya restringido a posición implosiva, pero con el tiempo haya extendido su contexto de aparición a posición prevocálica y final absoluta. En posición prevocálica el fenómeno está ya muy avanzado en el puertorriqueño.

El índice de conservación de la sibilante en posición prevocálica sería aún más bajo si no fuera por una tendencia fuerte a la conservación de $[\mathrm{s}]$ si le sigue una vocal tónica. Por ejemplo, si comparamos la conservación ante vocal tónica $(44 \%)$ con la conservación ante vocal átona $(12 \%)$, vemos que la diferencia es importante. Lo que es más, la conservación ante vocal tónica parece restringirse casi en su totalidad a la /s/ plural de determinativos: los otros, mis hijos, los únicos. En este contexto la frecuencia de la sibilante es muy alto: $85 \%$. No sucede así en el caso de pronombre más vocal tónica: ellos iban (11\%); o verbos más vocal tónica, es uno $(25 \%)$; etc.

En resumen, parece que la selección entre sibilante y aspirạción está condicionada casi exclusivamente por el contexto fonológico y que el puertorriqueño favorece la aspiración excepto en el caso de un determinativo más una vocal tónica (los únicos) y cuando se encuentra la /s/ en posición final absoluta.

\section{ESTUdIOS PREVIOS}

En su magistral estudio sobre el español de Puerto Rico, Navarro Tomás presenta una descripción detallada de las posibles variantes fonéticas encontradas por él. Para la posición implosiva -final de sílaba o de palabra- encontró que la aspiración era lo normal en "toda clase de personas y lugares, lo' mismo en las tierras altas que en las bajas y de igual modo en los centros importantes de población que en los más retirados barrios rurales" (op. cit., p. 71). Además, notó como posibilidad la asimilación completa o parcial a la consonante siguiente: avispa [abippa], los brazos [lob brasoh]. En posición final ante pausa, "la $s$ puertorriqueña desaparece normalmente en el habla popular [...]; la supresión de la $s$ final, extendida por toda la isla, se oye hasta en el habla familiar de las personas instruidas". En la presente investigación del habla culta 
las tendencias más importantes fueron: la conservación como sibilante (se articula en $40 \%$ del total) y la elisión de /s/ (se elidió en un $33 \%$ del total). Es posible que la diferencia entre la afirmación de Navarro Tomás y nuestros resultados se derive de la diferencia entre las clases instruidas y las de un nivel educativo menos avanzado.

Navarro Tomás dice también que "las dos primeras condiciones en que el campesino se apoya para elevar la corrección de su lenguaje son la reposición de la $d$ intervocálica y la de la $s$ sibilante". Esta afirmación parece indicar que Navarro Tomás considera la sibilante en posición final absoluta como señal de la ultracorrección. Los datos aquí presentados indican, sin embargo, que para la clase culta la variabilidad de $s \sim h \sim \phi$ es inherente al sistema mismo. En el caso de la posición prevocálica, que Navarro Tomás denomina "enlace sintáctico" aparece la alternancia de las tres variantes principales. Al no contar Navarro con los recursos de computación que usamos para este estudio, le fue imposible deducir la variabilidad sistemática correlacionada con el acento que nosotros encontramos.

El profesor Rubén del Rosario ${ }^{15}$ propone una descripción general de la aspiración de la /s/ para el área de Las Antillas. Según él, "se admite la pronunciación ocasional de esta consonante, sobre todo en la lectura en voz alta (cf. los resultados de Ma y Herasimchuk), pero no su exageración". Añade que "el arrastre de las eses tiene un fondo de vanidad: el hablante, inconscientemente, quiere mostrarse distinto o superior a su auditorio". El profesor del Rosario nota que la reposición de la /s/ se hace "ocasionalmente, sin regla fija". Dice, además, que "se conserva la /s/ en palabras como revés, nariz, solaz, tez" (la que hemos denominado aquí léxica). En este estudio no encontramos evidencia que apoyen estas afirmaciones.

En su análisis estructural del español puertorriqueño, Matluck ${ }^{16}$ trata de organizar los hechos en un esquema de distribución complementaria. Afirma que ocurre [h] ante consonante en posición interior y $\phi$ en final absoluta. Añade que /s/ "sólo recobra su aspiración en fonética sintáctica (ante vocal) cuando no sigue una pausa: dos y dos [doh i do].

En resumen, se ve que las observaciones efectuadas en estudios previos no dejan de tener validez, la que, por otra parte, dista mucho de ser absoluta. Tanto la carencia de materiales grabados como la ausencia del concepto de la variabilidad sistemática dentro de la

15 Rubén del Rosario, El español de América, Connecticut, 1970.

16 Josepil Matluck, "Fonemas finales en el consonantismo puertorriqueño", NRFH, 15 (1961), 332-342. 
teoría estructural provocaron una situación en la que no era posible hacer avances significativos en este terreno.

\section{Conclusiones e investigaciones futuras}

a) La aspiración y elisión de /s/ implosiva y final en el español de la clase culta de Puerto Rico es sistemática y se sujeta a factores condicionantes regulares.

b) La selección entre aspiración y sibilancia está regida principalmente por el contexto fonológico; los contextos pertinentes (ordenados según favorecen la sibilancia) son tres: ante pausa, prevocálico, preconsonántico.

c) Ante vocal tónica, la asibilación de /s/ es casi categórica en el caso de /s/ plural de determinativos y otros modificadores en primera posición en la frase nominal.

d) La elisión se rige por factores condicionantes funcionalesmorfológicos. En el caso de /s/ plural el puertorriqueño aplica la elisión de manera que conserve un morfema de pluralidad en la frase nominal en el adjetivo en primera posición. El único factor de importancia para la elisión en los demás casos (/s/ léxica $0 / \mathrm{s}$ / verbal) es el número de sílabas: la elisión es menos frecuente en el caso de monosílabos.

En el español puertorriqueño falta determinar los límites de la variación individual, la variación estilística, y la variación entre grupos sociales.

El segundo paso lógico sería hacer un estudio comparativo detallado de estos dos procesos fonológicos en las otras variedades del español del Caribe. Dadas las investigaciones realizadas sobre el español cubano y panameño, se espera que este tipo de estudio comparativo-dialectal se inicie en un futuro no muy distante. Más difícil, pero de sumo interés, será comparar el fenómeno y su alcance en todo el mundo hispánico, incluyendo el español de Chile, Argentina, Paraguay, Uruguay, Andalucía, y de otras áreas donde se producen normalmente los procesos de aspiración y elisión. Con esa base debe ser posible reconstruir el desarrollo diacrónico de esos fenómenos en el idioma español.

University of California, Irvine.

Tracy D. Terrell 